Louisiana State University

LSU Digital Commons

Faculty Publications

Department of Physics \& Astronomy

$12-20-2004$

\title{
MEGA: The next generation medium energy gamma-ray telescope
}

\author{
J. M. Ryan \\ University of New Hampshire Durham \\ R. Andritschke \\ Max Planck Institute for Extraterrestrial Physics \\ P. Bloser \\ NASA Goddard Space Flight Center \\ J. Cravens \\ Southwest Research Institute \\ M. Cherry \\ Louisiana State University
}

See next page for additional authors

Follow this and additional works at: https://digitalcommons.Isu.edu/physics_astronomy_pubs

\section{Recommended Citation}

Ryan, J., Andritschke, R., Bloser, P., Cravens, J., Cherry, M., Dicocco, G., Guzik, T., Hartmann, D., Hunter, S., Kanbach, G., Kippen, R., Kurfess, J., Macri, J., McConnell, M., Miller, R., Paciesas, W., Phlips, B., Regiero, V., Stacy, J., Strickman, M., Vestrand, W., Wefel, J., Wulf, E., Zoglauer, A., \& Zych, A. (2004). MEGA: The next generation medium energy gamma-ray telescope. Proceedings of SPIE - The International Society for Optical Engineering, 5488 (PART 2), 977-988. https://doi.org/10.1117/12.551891

This Conference Proceeding is brought to you for free and open access by the Department of Physics \& Astronomy at LSU Digital Commons. It has been accepted for inclusion in Faculty Publications by an authorized administrator of LSU Digital Commons. For more information, please contact ir@lsu.edu. 


\section{Authors}

J. M. Ryan, R. Andritschke, P. Bloser, J. Cravens, M. Cherry, G. Dicocco, T. G. Guzik, D. H. Hartmann, S. Hunter, G. Kanbach, R. M. Kippen, J. Kurfess, J. R. Macri, M. L. McConnell, R. S. Miller, W. Paciesas, B.

Phlips, V. Regiero, J. G. Stacy, M. Strickman, W. T. Vestrand, J. P. Wefel, E. Wulf, A. Zoglauer, and A. D. Zych 
University of New Hampshire

University of New Hampshire Scholars' Repository

Space Science Center

Institute for the Study of Earth, Oceans, and

Space (EOS)

10-11-2004

\section{MEGA: the next generation Medium Energy Gamma-ray Telescope}

James M. Ryan

University of New Hampshire, James.Ryan@unh.edu

R Andritsche

Max-Planck-Institut für extraterrestrische Physik

Peter F. Bloser

University of New Hampshire, Peter.Bloser@unh.edu

J Cravens

Southwest Research Institute

M L. Cherry

Louisiana State University - Baton Rouge

See next page for additional authors

Follow this and additional works at: https://scholars.unh.edu/ssc

Part of the Astrophysics and Astronomy Commons

\section{Recommended Citation}

James M. Ryan ; Robert Andritschke ; Peter F. Bloser ; James P. Cravens ; Michael L. Cherry ; Guido Di Cocco ; T. G. Guzik ; Dieter H. Hartmann ; Stanley H. Hunter ; Gottfried Kanbach ; R. M. Kippen ; James Kurfess ; John R. Macri ; Mark L. McConnell ; Richard S. Miller ; William S. Paciesas ; Bernard Phlips ; Victor Reglero ; J. G. Stacy ; Mark Strickman ; W. Thomas Vestrand ; John P. Wefel ; Eric Wulf ; Andreas Zoglauer and Allen D. Zych "MEGA: the next generation Medium Energy Gamma-ray Telescope", Proc. SPIE 5488, UV and Gamma-Ray Space Telescope Systems, 977 (October 11, 2004); doi:10.1117/

12.551891; http://dx.doi.org/10.1117/12.551891

This Conference Proceeding is brought to you for free and open access by the Institute for the Study of Earth, Oceans, and Space (EOS) at University of New Hampshire Scholars' Repository. It has been accepted for inclusion in Space Science Center by an authorized administrator of University of New Hampshire Scholars' Repository. For more information, please contact Scholarly.Communication@unh.edu. 


\section{Authors}

James M. Ryan, R Andritsche, Peter F. Bloser, J Cravens, M L. Cherry, G DiCocco, T G. Guzik, Dieter H. Hartman, Stanley D. Hunter, G Kanbach, R M. Kippen, J Kurfess, John R. Macri, Mark L. McConnell, R S. Miller, W Paciesas, B F. Phlips, V Reglero, J G. Stacy, Mark Strickman, W T. Vestrand, J P. Wefel, Eric Wulf, A Zoglauer, and Allen Zych 


\title{
MEGA: the next generation Medium Energy Gamma-ray Telescope
}

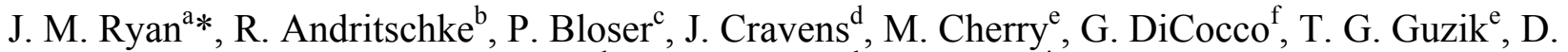 \\ H. Hartmann ${ }^{\text {g }}$, S. Hunter ${ }^{\mathrm{c}}$, G. Kanbach ${ }^{\mathrm{b}}$, R. M. Kippen ${ }^{\text {h, J. Kurfess }}{ }^{\mathrm{i}}$, J. R. Macri ${ }^{\mathrm{a}}$, M. L. McConnell ${ }^{\mathrm{a}}$,

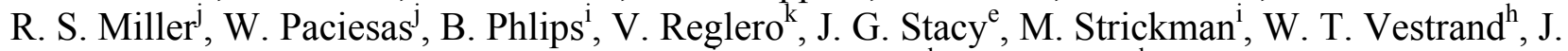 \\ P. Wefel ${ }^{\mathrm{e}}$, E. Wulf ${ }^{\mathrm{i}}$, A. Zoglauer ${ }^{\mathrm{b}}$, A. D. Zych';
}

\author{
${ }^{a}$ Space Science Center, Univ. of New Hampshire, Durham, NH, USA; \\ ${ }^{b}$ Max-Planck Institut für extraterrestrische Physik, Garching, Germany; \\ ${ }^{\mathrm{c}}$ Goddard Space Flight Center, NASA, Greenbelt, MD, USA: \\ ${ }^{\mathrm{d}}$ Southwest Research Institute, San Antonio, TX, USA; \\ ${ }^{e}$ Physics Dept, Louisiana State Univ., Baton Rouge, LA, USA; \\ ${ }^{\mathrm{f}} \mathrm{IASF} / \mathrm{CNR}$, Bologna, Italy \\ ${ }^{\text {g}}$ Physics Dept., Clemson Univ., Clemson, SC, USA; \\ ${ }^{\mathrm{h}}$ Los Alamos National Lab., Los Alamos, NM, USA; \\ ${ }^{\mathrm{i}}$ Naval Research Lab., Washington, DC, USA; \\ ${ }^{j}$ Physics Dept., Univ. of Alabama in Huntsville, Huntsville, AL, USA; \\ ${ }^{\mathrm{k}} \mathrm{GACE}$, University of Valencia, Spain \\ ${ }^{1}$ IGPP, Univ. of California/Riverside, CA, USA;
}

\begin{abstract}
The MEGA mission would enable a sensitive all-sky survey of the medium-energy $\gamma$-ray sky $(0.3-50 \mathrm{MeV})$. This mission will bridge the huge sensitivity gap between the COMPTEL and OSSE experiments on the Compton Gamma Ray Observatory, the SPI and IBIS instruments on INTEGRAL and the visionary ACT mission. It will, among other things, serve to compile a much larger catalog of sources in this energy range, perform far deeper searches for supernovae, better measure the galactic continuum emission as well as identify the components of the cosmic diffuse emission. The large field of view will allow MEGA to continuously monitor the sky for transient and variable sources. It will accomplish these goals with a stack of Si-strip detector (SSD) planes surrounded by a dense high-Z calorimeter. At lower photon energies (below $\sim 30 \mathrm{MeV}$ ), the design is sensitive to Compton interactions, with the SSD system serving as a scattering medium that also detects and measures the Compton recoil energy deposit. If the energy of the recoil electron is sufficiently high $(>2 \mathrm{MeV})$, the track of the recoil electron can also be defined. At higher photon energies (above $\sim 10 \mathrm{MeV}$ ), the design is sensitive to pair production events, with the SSD system measuring the tracks of the electron and positron. We will discuss the various types of event signatures in detail and describe the advantages of this design over previous Compton telescope designs. Effective area, sensitivity and resolving power estimates are also presented along with simulations of expected scientific results and beam calibration results from the prototype instrument.
\end{abstract}

Keywords: gamma rays, astrophysics, Compton telescope, satellite mission, silicon strip detector, scintillator

\section{INTRODUCTION}

Astronomy with low to medium energy $\gamma$ rays has always been an experimental challenge for two main reasons: (1) photon interaction cross-sections in the $\mathrm{MeV}$ range go through a minimum in their transition from the photoelectric effect $(\sim 100 \mathrm{keV})$ to pair creation $(\sim 10 \mathrm{MeV})$. In this so-called Compton range the interactions are characterized by small energy deposits and long-range secondary radiation. It is therefore necessary to build a "deep" detector to achieve a reasonable efficiency and to finely segment the detector in order to record and trigger widely separated interactions of the scattered photons, (2) the nuclear energy levels of all detector and structural materials lie in the $\mathrm{MeV}$ range and are therefore easily excited by energetic particles. The result of this systematic "radio-activation" is a prolific $\gamma$-ray 
background of local origin that must be effectively discriminated against to achieve a useful sensitivity for astronomical targets. COMPTEL was the first instrument in space to explore this difficult spectral band [1]. The results from COMPTEL proved that a wide variety of astrophysical sources and processes can be studied best in the range from $\sim 1$ $\mathrm{MeV}$ to several 10s of MeV. Now the third generation of pair-creation imaging telescopes (GLAST after SAS-2/COS$\mathrm{B}$ and EGRET) is scheduled to fly in 2006. It is time to develop also the second-generation telescope in the Compton range to maintain the overall multi-wavelength sensitivity that is so important for a full understanding of high-energy sources.

A mission that complements the anticipated scientific return of GLAST in the medium-energy range has been envisioned by NASA and dubbed the Advanced Compton Telescope, ACT. It is a visionary mission that exists in a conceptual stage of development. The technologies necessary to effect the improvement in sensitivity to compete with GLAST are being reviewed and evaluated at the time of this writing. Fig. 1 shows the point source sensitivity of some past, present and future instruments in the low through high-energy regimes. The lack of a sensitive instrument from 1$10 \mathrm{MeV}$ is evident. The COMPTEL and INTEGRAL instruments exhibit comparable sensitivities but fall well above the sensitivities at higher and lower energies. This is unfortunate since the emission physics of sources in this energy range is typically different than those in the neighboring energies making a host of interesting astrophysical objects too dim to be detected or investigated with current experiments. One fully expects the Universe to be as rich in sources at 1 $\mathrm{MeV}$ as it is at $10 \mathrm{keV}$ and $1 \mathrm{GeV}$, exhibiting the workings of the Universe at energies that are the most penetrating and revealing in the spectrum. The demand for a second-generation survey instrument is clear. A survey of the sky at a sensitivity that repeats what EGRET performed at $100 \mathrm{MeV}$ will highlight new objects that will be targets of ACT. At the same time, $\mathrm{MeV}$ objects that are or were merely detected with COMPTEL or INTEGRAL can now be studied quantitatively and in some detail.

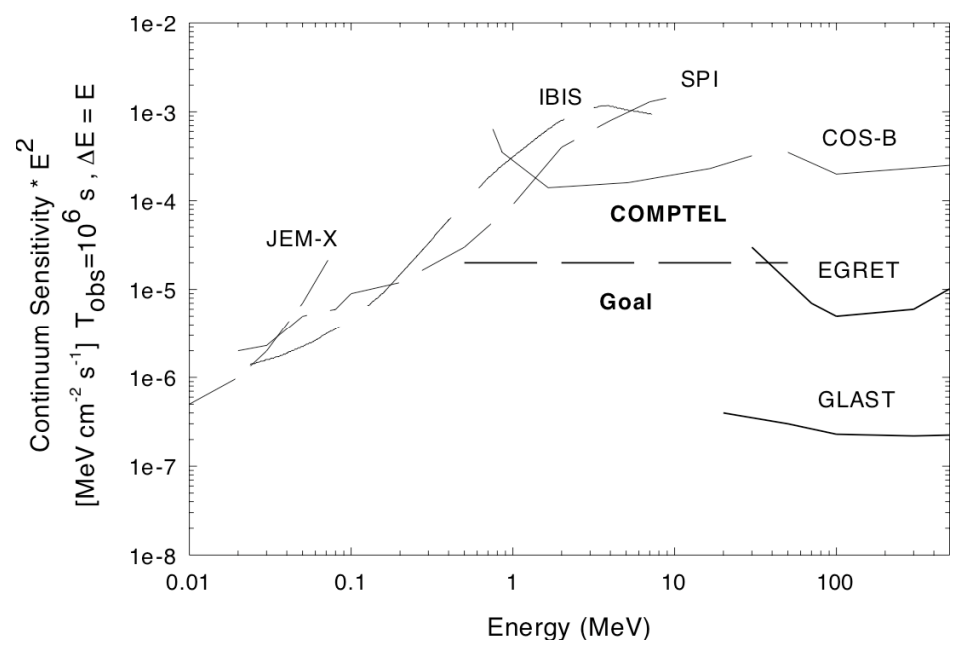

Fig. 1. Sensitivity curves for recent and current $\gamma$-ray and X-ray telescopes.

The target sensitivity for the next MeV spacecraft mission should fall half way, on a logarithmic scale, between the COMPTEL/INTEGRAL sensitivities and the extension of the GLAST sensitivity into the MeV range. A mission that attains this level of sensitivity will have another benefit, that being it will be achievable long before ACT is realized. It will provide a database for astrophysicists to examine and mine. It will sustain interest in this unique band of the electromagnetic spectrum. It will likely revolutionize $\mathrm{MeV}$ astronomy in the manner that EGRET revolutionized astronomy at $100 \mathrm{MeV}[2]$.

The huge gap in sensitivity at $\mathrm{MeV}$ energies is not for lack of trying. Detecting and measuring $\mathrm{MeV} \gamma$ rays is notoriously difficult. The mean free path of photons in this range is the longest in the field of high-energy astrophysics, meaning that they are the most difficult photons to stop and measure, while they can reach earth from the greatest reaches of the Universe with little attenuation or degradation. Compton scatters, the most efficient detection channel, 
only partially measures the photon energy, meaning multiple interactions are necessary when using this channel in an instrument. Nuclear reactions that fall in the $\mathrm{MeV}$ range make measurements here most important because such observations tell us about astrophysical nuclear processes, e.g., the origin of the elements. However, secondary cosmicray nuclear reactions, in particular those produced by neutrons, in and around a Compton telescope bathe any such instrument in copious $\mathrm{MeV}$ photons, washing out the signals from all but the brightest cosmic sources. So, the low probability of detection, the partial energy measurement and the cosmic-ray induced fog severely limit any instrument in this range.

A new mission must not only have greater effective area to accept and register more $\gamma$ rays, but it must retain or improve upon the rejection of background. To attain a larger effective area than COMPTEL need not mean a physically larger or more massive experiment. The COMPTEL effective area was small in part because of the small solid angle that the second detecting element D2 presented to the first detecting element D1. By bringing these two detecting elements closer together, this solid angle factor can be markedly increased, by as much as $10 x$.

However, moving these detectors closer together comes at a cost. COMPTEL employed time of flight as a means to reject background. At the small distances necessary for a $10 \times$ increase in solid angle, time of flight becomes problematic. Another method for rejecting background is necessary. Below we describe how MEGA provides a dramatic improvement in effective area while retaining the background rejection power that COMPTEL enjoyed.

While accomplishing a new sky survey intended to open up new investigations of the $\gamma$-ray sky, specific and targeted science can be addressed on issues that COMPTEL and other $\gamma$-ray instruments have left unfinished. These include the following:

- Search for and measurement of supernova emissions to better understand the dynamics of the explosion process and the creation of the elements. The anomalous redshift velocities of distant Type Ia supernovae have led to a critical review of the value of the cosmological constant. It is important to fully understand the Type Ia process since it is used here as a standard candle upon which we base a rethinking of the evolution of the Universe. COMPTEL was able to place upper limits on ${ }^{56} \mathrm{Co}$ emission from one supernova and marginally measure another. With a factor of ten improvement in sensitivity, it is likely that a supernova, in particular a Type Ia, will occur within a radius where good measurements can be conducted. These measurements would test our models of the phenomenon.

- Measurements of radioactive decay line emission from supernova remnants. Improving on the ${ }^{44} \mathrm{Ti}$ luminosity measurements by Iyudin et al. of Cas-A [3] would come along with luminosity measurements of ${ }^{26} \mathrm{Al}$ and ${ }^{60} \mathrm{Fe}$. These isotopes drive the last phases of the supernova expansion and populate the Galaxy with radioactive ash.

- The isotope ${ }^{26} \mathrm{Al}$ populates the plane of the Galaxy producing a diffuse Galactic emission. It was mapped by Diehl et al. but higher definition versions of these maps are necessary to properly associate the emission with distinct galactic structures.

- Black-hole candidates and AGNs exhibit emission in the MeV range, probably from the re-energization of $\mathrm{X}$ rays by scattering off high-energy electrons. A MeV tail on the spectrum of Cyg X-1 was discovered by McConnell et al. [4] and represents the kind of exciting physics that can emerge when studying such objects. AGNs have been associated with emission above $100 \mathrm{MeV}$, but this is likely due to the limited sensitivity that exists in the $\mathrm{MeV}$ band. It is likely that a broader picture of AGNs will emerge when some of them are measured in the $\mathrm{MeV}$ band. The object $3 \mathrm{C} 273$ is a rare example of an AGN that is luminous in $\mathrm{MeV} \gamma$ rays.

- It is also likely that a classical nova will occur during the MEGA mission. Classical novae produce other isotopes that radiate in the $\mathrm{MeV}$ range that again will constraint the dynamics of the process and the source function for lighter elements.

- Pulsars should be common in the Galaxy if one has the sensitivity to detect them. COMPTEL detected a handful of them, all displaying different behaviors in spectrum, light curve and frequency. This diversity makes categorizing them difficult. A larger set of $\gamma$-ray pulsars might exhibit trends and groupings that could be used to understand them. 
- Gamma-ray bursts exhibit some of their most interesting features at energies higher than the classical 100$200 \mathrm{keV}$. They have persisted for time scales of an hour at $\mathrm{GeV}$ energies [5], but their long-term behavior at $\mathrm{MeV}$ energies has been limited by sensitivity.

- The spectrum of the cosmic diffuse emission appears from research on COMPTEL, EGRET and SMM data to be comprised of several components. These components include active galaxies and supernovae. The supernova component is the least resolved and understood. MEGA would greatly improve on the COMPTEL measurements of this spectrum from below $1 \mathrm{MeV}$ to $50 \mathrm{MeV}$.

- Solar Flares exhibit the most complex spectrum in the sky. The richness of nuclear lines, bremsstrahlung emission, positron annihilation, and neutron capture contains much information about the process of accelerating the ions that produce these lines and the energy contained in these ions. Because of the complexity of the spectrum, the diagonal instrument response of a Compton telescope will greatly aid in the unambiguous interpretation of the data.

To accomplish these goals with a medium sized mission requires new designs beyond that of COMPTEL. We have been developing and testing such a design for a new Compton telescope. It functions in some ways similar to COMPTEL, but differs in many important aspects.

COMPTEL used liquid scintillator (D1) as the scattering medium, while MEGA uses silicon in the form of silicon strip detectors (SSD). The SSDs are thin and a stack of them presents a target for the incoming $\gamma$ ray. By using thin detectors, one can register the recoil Compton electron in more than one unit. This allows one to construct a recoilelectron momentum vector.

COMPTEL used NaI scintillator as the second or stopping detector. MEGA, in its prototype form, uses CsI, but in much smaller units than the COMPTEL D2 NaI detectors.

COMPTEL had a separation of $1.5 \mathrm{~m}$ between D1 and D2, while that distance has shrunk to about $30 \mathrm{~cm}$ on average for MEGA since the D2 detector will surround the D1 detector on five sides of a cube, leaving only the top of the cube as the instrument aperture. The use of SSDs and the close spacing of D1 and D2 in MEGA preclude the use of time of flight as a background discriminator. This is the greatest value for tracking the recoil electron in the SSDs, that is, it serves to effectively reject background. A schematic of the basic MEGA design is shown in Fig. 2.

Below we discuss the implementation of this design in a prototype, its measured properties, our plans for testing the unit and the possible deployment of a mission based on this concept on a medium-sized mission.

\section{MEGA PROTOTYPE}

\subsection{Principal of Operation}

Before the development of a spacecraft instrument it is essential that the design and technology be tested in a realistic environment. The best and least expensive route to follow is a build a prototype that employs all the critical technology that would be necessary for space, calibrate it in a laboratory and fly it on a balloon platform. These measurements and tests should be accompanied by extensive simulations, not only for effective area and instrument response, but for background susceptibility as well. Here we describe the MEGA prototype instrument and the result of testing and calibration.

Compton telescopes operate on the principle that Compton scattering is the dominant reaction in an instrument. If one could trace the history of scattering within an instrument then the incident direction and energy of the $\gamma$ ray could be determined through the Compton formula

$$
\cos \varphi=1+\frac{m_{e} c^{2}}{E_{\gamma}}-\frac{m_{e} c^{2}}{E_{\gamma}-E_{1}},
$$

where $\varphi$ is the scatter angle of the photon, $E_{\gamma}$ is the photon energy and $E_{1}$ is the energy deposited to the recoil electron. With a complete measure of $E_{\gamma}$ and a measure of the recoil-electron momentum vector, one has the complete information about the incident $\gamma$ ray. With less than this one can only restrict or constrain with photon energy or its 
incident direction. Without measuring the recoil-electron direction the incident direction is restricted to the mantle of a cone whose axis is the direction of the scattered $\gamma$ ray as shown in Fig. 2.

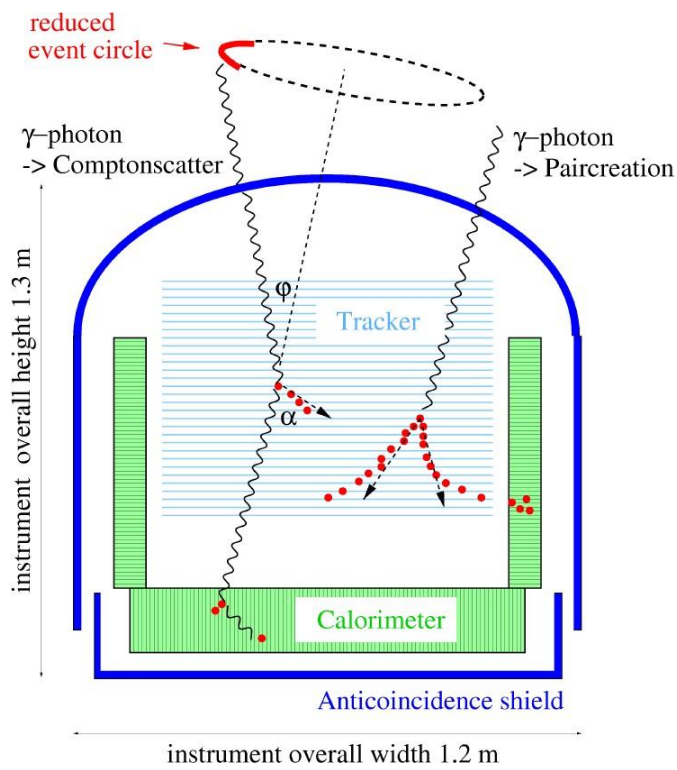

Fig. 2. Schematic of the MEGA instrument and kinematics of $\gamma$-ray interactions.

Two physical processes dominate the interaction of photons with matter in the medium-energy $\gamma$-ray band: Compton scattering at low energies, and electron-positron pair production at high energies, with the changeover at 5-10 MeV for most detector materials. In both cases the primary interaction produces long-range secondaries whose directions and energies must be determined in order to reconstruct the incident photon. MEGA, like previous Compton and pair creation telescopes, will employ two separate detectors to accomplish this task: a tracker, in which the initial Compton scatter or pair conversion takes place, and a calorimeter, which absorbs and measures the energy of the secondaries (see Fig. 2). In the case of Compton interactions, the incident photon scatters off an electron in the tracker. The interaction position and the energy imparted to the electron are measured. The scattered photon interaction point and energy are recorded in the calorimeter. From the positions and energies of the two interactions the incident photon angle $\varphi$ is computed from the Compton equation, Eq. 1. The primary-photon incident direction is then constrained to an event circle on the sky. For incident energies above about $2 \mathrm{MeV}$ the recoil electron usually receives enough energy to penetrate several layers, allowing it to be tracked. This further constrains the incident direction of the photon (the reduced event circle in Fig. 2).

The differential Klein-Nishina cross section for Compton scattering contains a strong dependence on the polarization of the incident $\gamma$-ray photon. Scattered photons are emitted preferentially perpendicular to the direction of the electric field vector of the incoming photon. The strongest azimuthal modulation in the distribution of scattered photons will be for $\gamma$-ray energies between $1-5 \mathrm{MeV}$ and scatter angles of $20^{\circ}-60^{\circ}$. This will make a Compton telescope with a calorimeter covering a large solid angle a unique polarimeter for $\gamma$ radiation. In the case of pair production, the incident photon converts into an electron-positron pair in the tracker. These two particles are tracked and determine the incident photon direction. The total energy is then measured through the deposits absorbed in the tracker and/or the calorimeter.

The design of a new high-energy $\gamma$-ray telescope must be based on numerical simulations as well as experimental detector developments. From the concept in Fig. 2 we used GEANT3 to develop the baseline for a satellite version of MEGA. In this version, the tracker contains 32 layers of double-sided Si detectors (thickness $500 \mu \mathrm{m}$, area $6 \times 6 \mathrm{~cm}^{2}$ each wafer, $6 \times 6$ wafers/layer). The pixelated CsI calorimeter is $8 \mathrm{~cm}$ deep on the bottom and $4 \mathrm{~cm}$ deep on the sidewalls. The cross-section of each CsI element is $5 \times 5 \mathrm{~mm}^{2}$. These choices also motivated the decision to construct a representative prototype detector (Fig. 3) of about $25 \%$ of the area and $33 \%$ of the depth of the full satellite version. This prototype has been used to evaluate the performance characteristics in a series of calibrations. In the following 
sections we describe the telescope components in more detail and present their properties in current experimental results.

\subsection{MEGA components}

The tracker must accomplish several different tasks. It must: (1) act as the scattering medium for Compton interactions; (2) measure the scattering location and the energy imparted to the recoil electron; (3) act as the conversion medium for pair production; (4) provide a large interaction volume for both processes to achieve good sensitivity; (5) record the tracks and energy deposits of all secondary particles, both electron-positron pairs and Compton recoil electrons; (6) provide a fast timing signal to be used in a coincident trigger with the calorimeter; and (7) operate without elaborate cooling and with reasonable power consumption. Thus good position, energy, and time resolution are required simultaneously in a large volume. The logical choice of detector technology for the tracker was a stack of double-sided silicon strip detectors.

For the prototype instrument these detectors were designed by the MPE semiconductor laboratory and produced by Eurisys Mesures, Lingolsheim, France. The availability of low-noise, low-power multi-channel preamplifier chips makes it feasible to read out a large number of channels within the space and power constraints of a space mission, so that the scintillator technology of COMPTEL may be replaced with semiconductors. The use of strips allows large areas to be covered with a reasonable number of channels. Although silicon strip detectors have been used as particle trackers in accelerator experiments, the requirements of the MEGA tracker impose special demands, namely 2-d position information from each layer, good energy resolution, and low power consumption. Each layer of the tracker is composed of a $3 \times 3$ array of $500 \mu \mathrm{m}$ thick silicon wafers, each $6 \times 6 \mathrm{~cm}^{2}$ in size and fitted with 128 orthogonal $\mathrm{p}$ and $\mathrm{n}$ strips on opposite sides $(470 \mu \mathrm{m}$ pitch). The strips are biased using the punch-through principal and AC-coupled via metal strips separated from the strip implant by an insulating oxide/nitride layer. The strips from adjacent wafers in the $3 \times 3$ array are wire-bonded in series and read out by 128 -channel TA1.1 ASICs, creating a total area of $19 \times 19 \mathrm{~cm}^{2}$ position-sensitive area. At $20 \mathrm{C}$ a typical energy resolution of $15-20 \mathrm{keV} \mathrm{FWHM}$, a position resolution of $290 \mu \mathrm{m}$ (measured with muon tracks), and a time resolution of $\sim 1 \mu \mathrm{s}$ is measured. The stack in the MEGA prototype detector (Fig. 3) will contain at least 10 layers. A few extra layers of inferior quality (e.g. only one-sided readout) due to deficiencies in the Si wafers may become available and could be inserted as particle passage detectors. Fig. 3 also shows the front-end electronics boards mounted next to the Si wafers.

The tracker for the satellite size MEGA could be assembled from four quadrants, each similar to the prototype tracker, with the electronics on the outer rim. The next development for tracker technology will be the use of $10 \times 10 \mathrm{~cm}^{2} \mathrm{Si}$ wafers, which could lead to an even denser detector volume with less structural material.

The MEGA prototype calorimeter consists of 20 modules, each with an array of $10 \times 12 \mathrm{CsI}(\mathrm{Tl})$ scintillator bars of crosssection $5 \times 5 \mathrm{~mm}^{2}$ read out with Silicon PIN-diodes and low-noise, self-triggering front-end electronics. The length of the bars (the depth of the calorimeter) was chosen to correspond to the stopping power needed to absorb the scattered photons at different angles, i.e., large scatter angles produce low energy secondary photons that are stopped in thinner crystals while forward scattering requires a thicker calorimeter. The upper side modules are $2 \mathrm{~cm}$ deep, the lower side wall $4 \mathrm{~cm}$, and the bottom calorimeter is $8 \mathrm{~cm}$ deep. The CsI(Tl) bars were manufactured by Hilger Crystals and are polished on all sides. The calorimeter modules are packed with the CsI bars separated by several layers of reflective paper (millipore). The optical coupling between scintillators and photodiodes is through a prefabricated array of transparent silicon cushions where the individual pixels are separated by a grid of opaque white silicon. The $8 \mathrm{~cm}$ modules are read out from both ends. The ratio of the two signals allows the location of the energy deposit along the bar with a typical resolution of $\sim 2 \mathrm{~cm}(\mathrm{FWHM})$. This 3-d resolution, together with the size of the energy deposit, is important for reconstructing the correct sequence of multiple interactions in the calorimeter. The energy resolution of the calorimeter modules was measured with radioactive sources. Typical results for $\Delta \mathrm{E} / \mathrm{E}$ (FWHM) at the incident energy of $662 \mathrm{keV}$ range between $8 \%$ (for a $2 \mathrm{~cm}$ unit) and $10 \%$ (for $8 \mathrm{~cm}$ ). The energy resolution of the calorimeter is so far the limiting factor for the angular resolution of the MEGA design. Detectors with a decisively better resolution (e.g. segmented CZT or Ge) could very much improve the telescope imaging quality. A viable short-term development seems to be the application of new, low noise, Silicon drift detector diodes to replace the PIN diodes. This change could improve on the energy resolution by about a factor 2-3. 


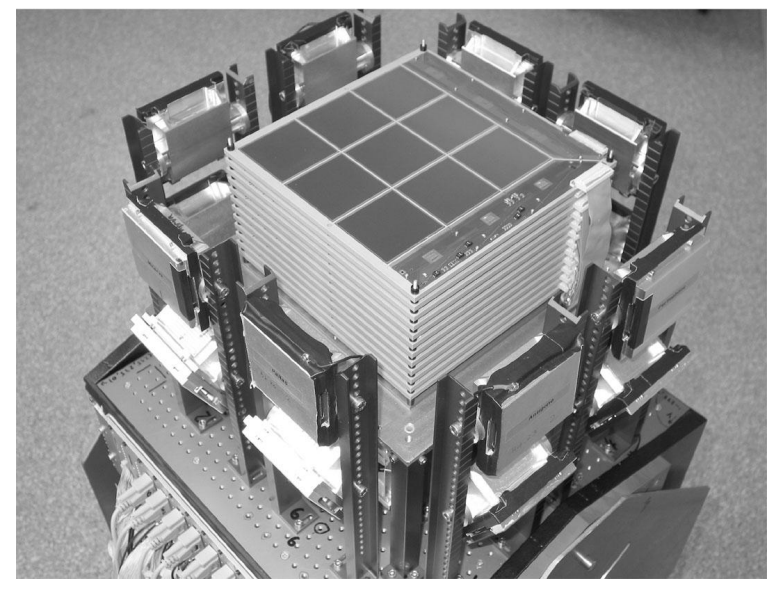

Fig. 3. The MEGA prototype including SSD and CsI detectors.

The MEGA prototype [6] contains 8448 and 2880 channels of measurement in the tracker and calorimeter respectively. A satellite telescope with the same pixelation contains more than 10x this number of data channels. It is evident that the data acquisition from this large number of channels demands highly integrated, low power electronics. For the prototype instrument the front-end, repeater card, opto-coupler, trigger processing card and power supplies for electronics and detectors are custom developments. The back-end with the signal transceivers, analog-to-digital converters, and an onboard computer (OBC) with mass storage (10 GB hard disk), is based on VME modules. The data acquisition and detector control software is based on $\mathrm{C}++$ and the ROOT software library from CERN. It is implemented and operates on the PC Linux system (Pentium III, $850 \mathrm{MHz}$ ) of the OBC. In the front-ends, of both the tracker and calorimeter, we use VLSI amphfier-ASICs of type TA1.1 designed by IDE with built-in trigger capability. These ASICs are well suited for the read-out of Si detector signals and have the following characteristics: (1) low noise level of $165 \mathrm{e}^{-}+7 \mathrm{e}^{-} / \mathrm{pf}$ with a $2 \mu$ s shaping time; (2) a dynamic range of $10 \mathrm{MIPs}$; (3) 128 channels in parallel with serial readout up to $10 \mathrm{MHz}$ clock rate; (4) low power consumption of $\sim 1.5 \mathrm{~mW} /$ channel (5) common trigger output from 128 level discriminators with common adjustable threshold; and (6) a 128 bit blocking register to prevent individual noisy discriminators from triggering.

The so-called repeater card supplies up to 3 front-end ASICs with regulated and precise power levels and manages the commanding of the threshold and trigger registers. When a system trigger has been generated the sequencer in the repeater card issues the necessary commands to sample and hold the measured signals. The sequential reading of all measurement channels is also initiated by the sequencer. Communication between the repeater and the back-end passes through an optocoupler to galvanically isolate repeater and front-end, which are on the bias levels of the Si detectors, from the rest of the system. The trigger processor unit checks for coincidences and combines the trigger signals from 21 segments of the system, i.e., up to 12 tracker layers, 8 groups of calorimeter units, and an anticoincidence signal. If no absolute veto signal has been received the trigger pattern, in the form of a 21-bit word, is used as an address to interrogate a 2 MB RAM where the valid trigger combinations are stored as non-zero bits. The flexibility to generate different memory loads for this trigger register allows one to select the best events and to adjust the trigger rate to an acceptable level. The trigger processor is implemented on a FPGA of type Xilinx-Spartan. The back-end contains a commercial VME system with modules for signal conditioning, analog to digital converters, generation of a GPS based time stamp, and the central processor with data storage on a hard disk.

\subsection{Calibrations}

From January to March 2003 the prototype was calibrated with laboratory sources $\left({ }^{22} \mathrm{Na},{ }^{137} \mathrm{Cs},{ }^{88} \mathrm{Y}\right)$ in the near field, and in April/May 2003 it was calibrated at the High Intensity Gamma Source HI S [7] at Duke University (Durham, North Carolina) in the far field. The latter calibration used monoenergetic $(\Delta \mathrm{E} / \mathrm{E}<2 \%)$ and $100 \%$ polarized pencil beams at different energies $(0.7,2,5,8,10,12,17,25,37$ and $49 \mathrm{MeV})$ and different incident angles $\left(0^{\circ}, 30^{\circ}, 60^{\circ}, 80^{\circ}\right.$, $120^{\circ}, 180^{\circ}$ ). Based on these data, preliminary imaging properties of the telescope have been determined. 
The image reconstruction is performed with an unbinned maximum-likelihood method called list-mode maximumlikelihood expectation maximization, which originally was developed for medical imaging of a SPECT camera [8] and later it was adapted for its use in astrophysics [9]. This method allows the incorporation of different event types (tracked and untracked Compton events as well as pair events) into one image while preserving all measured information.

\subsubsection{The Compton Scatter Regime \\ 2.3.1.1 Imaging Response.}

Ideally, a tracking Compton telescope should measure all parameters to directly calculate the origin of the incoming photon: direction and energy of the scattered photon as well as direction and energy of the recoil electron. Below $2 \mathrm{MeV}$ the energy of the electron is in most cases not sufficient to generate a track in D1, the SSD stack. For these events the origin of the photon can only be restricted to the classical cone section, which is represented by circles in the far field and ellipses/hyperbolas in the near field. Both lead to a large ambiguity of the origin of these photons. The width of this cone section is mainly determined by the energy measurement. On the other hand, even with the knowledge of the electron direction, the incident direction cannot be restricted to a single vector: Moliére (small-angle) scattering in the silicon produces a large uncertainty in the direction of the electron track. The knowledge of the cone section, determined by the measured energies, in combination with the electron track, restrict the possible origins of the photon to short arcs of the cone section, as seen in Fig. 4.

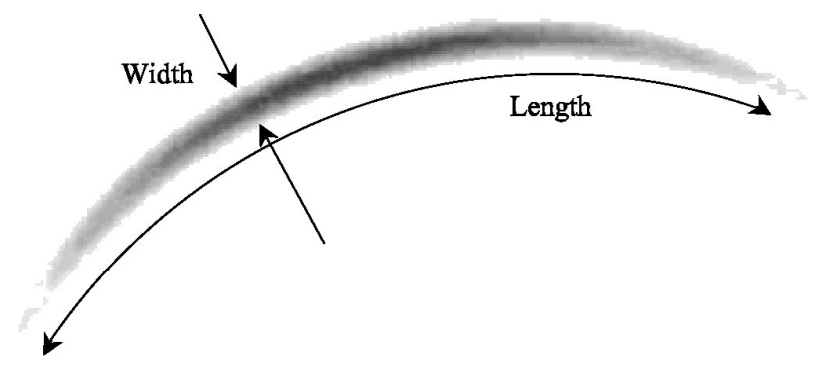

Fig. 4. The possible incident directions of tracked $\gamma$ rays are restricted to an arc.

\subsubsection{Angular Resolution.}

One method of characterizing the width of the response and therefore the angular resolution is the Angular Resolution Measure (ARM). It is defined as the difference between the real and the measured Compton scatter angle. An example is given in Fig. 5. This distribution contains tracked as well as untracked events at $2.0 \pm 0.2 \mathrm{MeV}$ and has a FWHM of $7.4^{\circ}$. If only untracked events are considered, the shape is narrower $\left(6.2^{\circ}\right)$, whereas tracked events produce a much broader ARM $\left(13.4^{\circ}\right)$. This behavior is expected, since tracked events suffer especially strongly from the limited energy resolution in the calorimeters: On the one hand tracks are only generated if sufficient energy is transferred to the electron. This results in lower energy deposits in the calorimeter and therefore a higher relative energy measurement error for the scattered $\gamma$ ray. On the other hand, the measurement error, which plays the most important role for determining the error in the Compton scatter angle $\varphi$ for large scatter angles, is the energy measurement error of the scattered $\gamma$ ray. With the current and still preliminary energy resolution, which ranges for example in the 8 -cm bottom calorimeters from $\Delta \mathrm{E} / \mathrm{E}=0.15$ to 0.22 at $662 \mathrm{keV}$, the angular resolution for tracked events is far away from the physical limit, which is given by Doppler-broadening. At $2 \mathrm{MeV}$ Doppler-broadening would lead to an average width of $\sim 0.2^{\circ}$ in silicon [9]. Therefore having a calorimeter with an excellent energy resolution is crucial for the performance of a tracking Compton telescope. The angular resolution as a function of the energy (Fig. 5) at lower energies is mainly determined by the measurement error of the energy. Going from lower to higher energies, the performance first improves and then deteriorates again, since incomplete absorptions start to dominate: The scattered photons leak and the electrons are no longer stopped in the tracker. Nevertheless, incomplete absorption will be much less pronounced in the larger satellite geometry with its more compact calorimeter. 


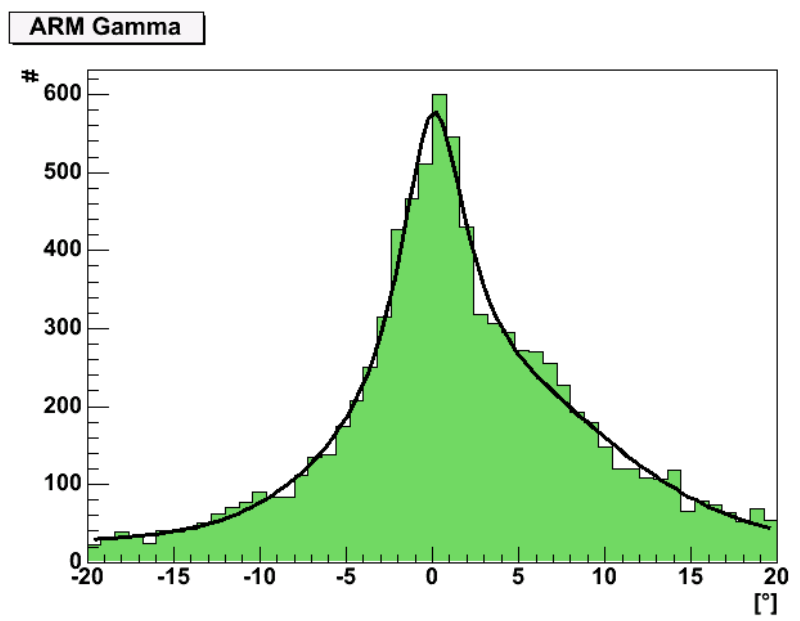

Fig. 5. Measured ARM distribution of tracked and untracked event from 1.8 to $2.2 \mathrm{MeV}$ with a FWHM of $\sim 7.4^{\circ}$.

\subsubsection{Direction of Electron Tracks.}

The most important characteristic of a tracking Compton telescope is the electron track. Using $\gamma$ rays from a $1.8 \mathrm{MeV}$ ${ }^{88} \mathrm{Y}$ source the HWHM of the track direction error distribution is $\sim 42^{\circ}$. This is close to the physical limit determined by Moliére scattering. At this relatively low energy more than $85 \%$ of all tracks can be reconstructed correctly within the limits stated above.

\subsubsection{Polarization.}

Most processes in high-energy astrophysics can generate polarized $\gamma$ radiation (e.g. synchrotron radiation, bremsstrahlung, Compton scattering, etc.). Polarization measurements are of great value in understanding the emission mechanisms of $\gamma$ rays. The Compton cross section is polarization-dependent and has its greatest signature at large scatter angles. MEGA with its geometry (see Fig. 2) is well suited to detect polarization. Fig. 6 shows such a polarization signal. The measurement was performed with $100 \%$ polarized $710 \mathrm{keV} \gamma$ rays produced at the HI $\gamma$ S facility at Duke University. The $\gamma$ rays where produced by the Inverse Compton process and are intrinsically polarized at the $100 \%$ level. This was a problem in attempting to measure the detector response to unpolarized $\gamma$ rays. An unpolarized beam is necessary to correct for the asymmetries of the instrument when measuring a polarized beam. So, to measure the response to an unpolarized bean we used a ${ }^{137} \mathrm{Cs}$ source $(662 \mathrm{keV})$ positioned one meter above the detector. Registered events with unphysical large energies were discarded from the analysis. The azimuth angle of the scattered photon is measured with each detected event. These azimuth angles are then histogrammed to search for a sinusoidal signal (after correcting for the detector asymmetries). The modulation factor $\mu$, which gives the degree of polarization, is defined as

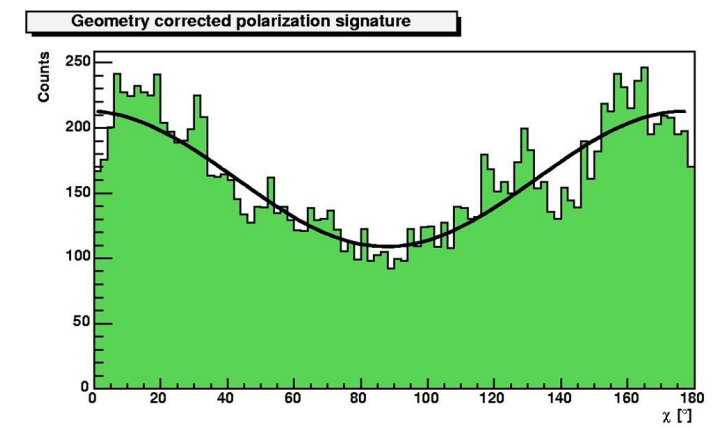

Fig. 6. Geometry corrected event distribution of the azimuth scatter angle.

$$
\mu=\frac{N_{\max }-N_{\min }}{N_{\max }+N_{\min }},
$$

where $N_{\max }$ and $N_{\min }$ are the number of counts at the maximum and the minimum of the azimuth angle distribution. From the $100 \%$ polarized beam of the Duke measurement this modulation factor was measured to be $\mu_{100}=0.31 \pm 0.03$ (Fig. 6). The error is statistical and $\mu$ was not corrected for any time-varying detector response. An additional correction for the divergent ${ }^{137} \mathrm{Cs}$ beam adjusts the value of $\mu_{100}{ }^{\text {corr }}=0.30 \pm 0.03$. Monte Carlo simulations predict a modulation of $\mu_{100}=0.304$. The geometry of the instrument/beam setup should produce a maximum at $0^{\circ}$, whereas the maximum was measured to be $-4.6^{\circ} \pm 2.1^{\circ}$. Other measurements at $2 \mathrm{MeV}$ with poorer statistics show a modulation of $\mu_{100}{ }^{\text {corr }}=0.13 \pm 0.04$, also in agreement with the simulations. 


\subsubsection{Multiple and Extended Sources.}

In a realistic astrophysical environment a telescope must detect and resolve multiple sources within the field of view. To test the instrument capability to do this we placed five sources of different energies with different intensities at different positions in the field of view of MEGA. All five sources (not shown) were reconstructed at the correct positions. It is also necessary to resolve extended sources such as supernova remnants or OB associations. This ability was demonstrated by a measurement, where two ${ }^{88} \mathrm{Y}$ sources were mounted on a rotating propeller located $27 \mathrm{~cm}$ above the center of the tracker. The reconstructed image is shown in Fig. 7. The sources moved in a circular path of radius $7 \mathrm{~cm}$. This corresponds to an angular diameter of $\sim 29^{\circ}$ at infinity. The image contains 138,000 Compton events in the energy range from 0.8 to $1.0 \mathrm{MeV}$. The non-uniformities of the image are artifacts of the non-uniformities of the instrument response, an effect that should be correctable.

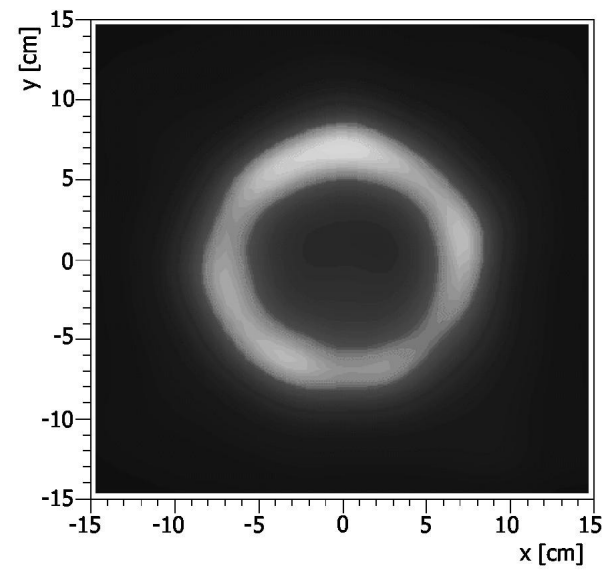

Fig. 7. The time-averaged image of two $\gamma$-ray sources moving in a circular path in the field of view.

\subsubsection{Pair-production regime}

In silicon the dominant reaction for $\gamma$ rays is pair production once the photon falls above $8 \mathrm{MeV}$. MEGA can track the electron-positron pair and determine the incident energy and direction.

\subsubsection{Angular Resolution.}

The angular resolution of pair events is dominated by the unmeasured recoil of the nucleus and electron Moliére scattering in the SSDs. The influence of both effects is greatest at threshold $(8 \mathrm{MeV})$ and diminishes thereafter as illustrated in Fig. 8 from calibration data. The angular resolution, given as the cone half-angle, which contains $68 \%$ of all events, is illustrated in Fig. 9. At $49 \mathrm{MeV}$ it is roughly $2 \times$ better than in the EGRET telescope. At energies below $100 \mathrm{MeV}$ it will also surpass the expected performance of GLAST and AGILE, because these telescopes contain passive converter foils that scatter the electrons.

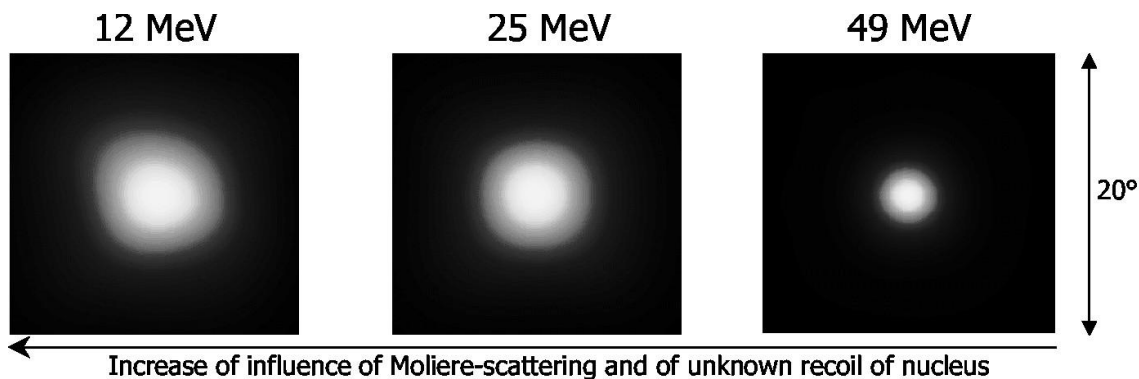

Fig. 8. Pictorial representation of the measured angular resolution in the pair mode. 


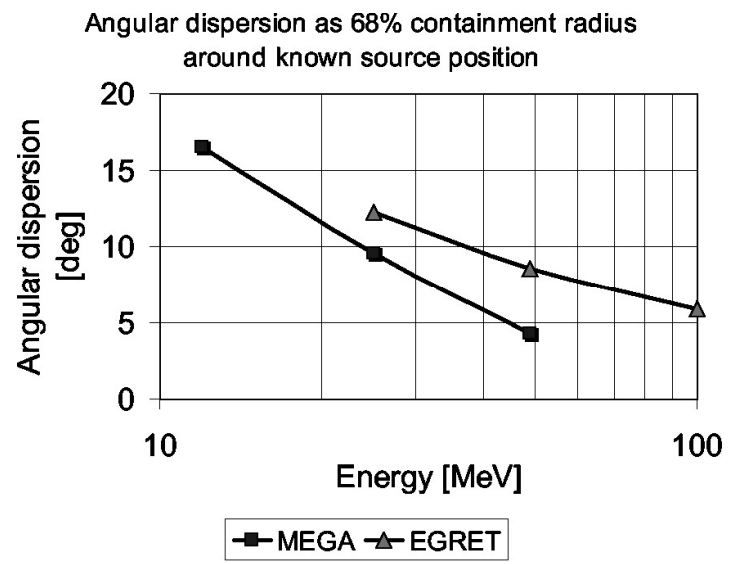

\subsubsection{Calibration Summary}

Fig. 9. Angular resolution curves for MEGA and EGRET.

This calibration demonstrated that the MEGA technique of detecting gamma rays works for a large energy range (from $500 \mathrm{keV}$ up to $49 \mathrm{MeV}$ and more) over a wide field of view. It also shows that MEGA can detect polarization up to at least $2 \mathrm{MeV}$, and that in the pair regime the telescope has excellent angular resolution.

Poorer angular resolution exists in the Compton regime, because it is dominated by the poor energy resolution of the calorimeters. However, an improved version of the calorimeter is under development to improve the energy resolution (at least $2 \times$ ) and lower the thresholds (at least $3 \times$ ). Simulations show, that with drift-diodes in the larger satellite geometry, with a more compact calorimeter, an angular resolution for tracked events of $\sim 2^{\circ}$ at $2 \mathrm{MeV}$ is achievable.

\section{BALLOON FLIGHT PREPARATION}

The prototype is being prepared for a high-altitude flight to measure the background in a high-background environment. We distinguish this part of the program by renaming the instrument MEGABALL. The MEGA prototype telescope is the core of the payload. It will be surrounded by an anticoincidence shield (ACS) (Fig. 10) to reject charged particles of cosmic and atmospheric origin. The ACS is made of $1.25-\mathrm{cm}$ plastic scintillator plates (Bicron BC 412). See Fig. 11. The scintillation light is measured by PMTs interfaced to wavelength shifting fibers that are optically coupled to the scintillator plates. The telescope and the front-end electronics, including the repeaters and optocouplers, are housed in a pressure vessel. A second pressure vessel encloses the back-end and command electronics. The attitude of the telescope is not actively controlled but is frequently and regularly measured $(\sim 0.5 \mathrm{~s})$ with a differential GPS. The GPS will also provide the absolute time to be imbedded in the event records. A detection of the Crab nebula will require at least 6-7 hours that may be beyond the flight opportunity. A balloon flight is planned for 2005 .

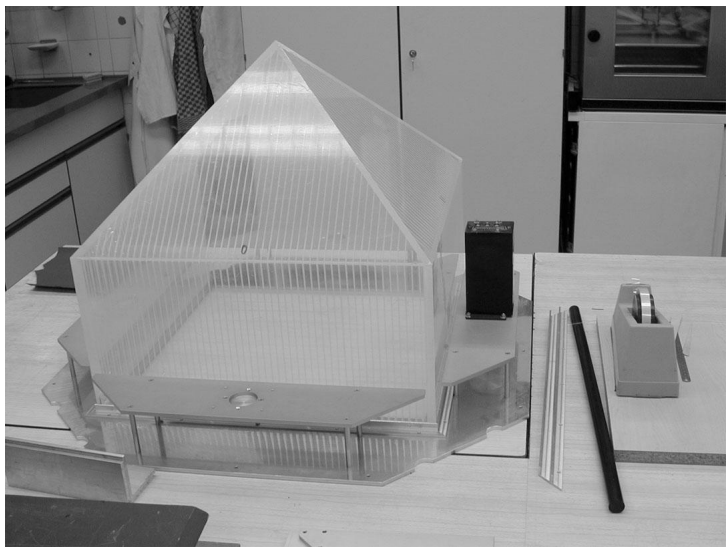

Fig. 10. The anticoincidence system of MEGABALL.

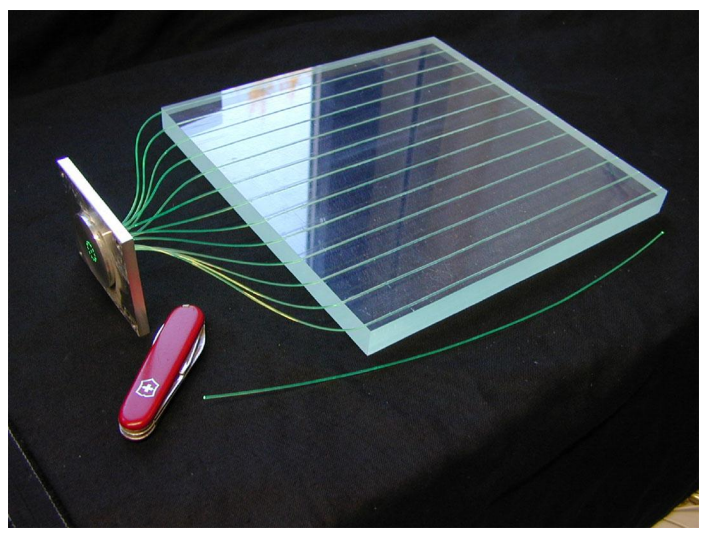

Fig. 11. A charged-particle shield detector outfitted with wavelength-shifting fibers 


\section{MEGA MISSION}

A mission built around a Compton telescope like MEGA should fit on within NASA MIDEX envelope in terms of mass, power and cost. The ideal orbit of the MEGA mission should be a variation of a zenith pointing equatorial orbit. The advantage of such an orbit is that the spacecraft and instrument avoid the South Atlantic Anomaly, greatly minimizing the activation of material in and around the instrument and the earth's atmosphere is the farthest from the instrument aperture as possible. Both internal activation and albedo $\gamma$ rays played major roles in limiting the sensitivity of COMPTEL. Except for the celestial poles, the sky will get complete coverage without the complexity of regular attitude control and pointing. Dedicated pointings for bursts and targets of opportunities may still be possible at the expense of complicating spacecraft operations and spacecraft complexity.

A two-year survey mission would re-invigorate and re-energize a rich field of astrophysics. It would build on the success of the Compton Observatory and the continuing success of INTEGRAL. However, by viewing the Universe deeper than before, it would bring to the surface exciting new phenomena.

What remains is a successful balloon flight and an assessment of the background sensitivity to prepare this instrument concept for a space mission.

\section{ACKNOWLEDGEMENTS}

We would like to acknowledge the institutional support of the University of New Hampshire, the Max-Planck Institute, Goddard Space Flight Center, the University of Alabama, Louisiana State University, the Naval Research Laboratory, IGPP, Clemson University, GACE and IASF/CNR.

\section{REFERENCES}

1. Schönfelder, V. et al., The GRO-COMPTEL Mission: Instrument Description and Scientific Objectives in Data Analysis in Astronomy, 1991, Erice, Sicily.

2. $\quad$ Kanbach, G., et al., The MEGA project, New Astronomy Reviews, 2004, 48(1-4): p. 275-280.

3. Iyudin, A.F. et al., COMPTEL observations of Ti-44 gamma-ray line emission from CAS A, Astronomy and Astrophysics, 1994, 284: p. L1-L4.

4. McConnell, M.L. et al., The Soft Gamma-Ray Spectral Variability of Cygnus X-1, Astrophysical Journal, 2002, 572(2): p. 984-995.

5. Dingus, B.L., EGRET Observations of $>30 \mathrm{MeV}$ Emission from the Brightest Bursts Detected by BATSE, Astrophysics and Space Science, 1995, 231: p. 187-190.

6. Andritschke, R. et al., The calibration setup of the MEGA prototype at the high intensity $\gamma$-ray source, New Astronomy Reviews, 2004, 48(1-4): p. 281-285.

7. Litvinenko, Vladimir N. et al., Short-wavelength light sources at Duke storage ring in Proceedings of SPIE: Electron-Beam Sources and Charged-Particle Optics, 1995: SPIE.

8. Flynn, Michael J., S. Wilderman, and J. Kanicki. Effect of secondary radiations on the performance of digital radiographic detectors in SPIE: Physics of Medical Imaging, 1998: SPIE.

9. Zoglauer, A., R. Andritschke, and G. Kanbach, Data analysis for the MEGA prototype, New Astronomy Reviews, 2004, 48(1-4): p. 231-235. 